\title{
Accomplishing Interconnectivity of Health Information Exchanges at the National and State Levels
}

\author{
Dalvin Hill ${ }^{1+}$ and David Devine ${ }^{2}$ \\ ${ }^{1}$ Stella and Charles Guttman Community College, USA \\ ${ }^{2}$ South University - Austin, TX, USA
}

\begin{abstract}
Health Information Exchanges (HIEs) are becoming more popular in the healthcare sector. This can be attributed to the unfolding benefits of connecting HIEs together, thus fostering the sharing of patient data. There are areas of improvement and this paper aims to interconnect the various existing and future HIEs that are used to capture, transmit, and store electronic health records. This paper aims to accomplish interconnectivity by combining the efforts of Patient Access Number (PAN) with the Federal Bureau of Investigation's implementation of the National Crime Information Center. By combining both efforts, each patient will have a unique identifier, i.e. a PAN, which can be used to search for a patient's health record both on a local/state and national level. This interconnectivity can lead to another step in the Meaningful Use ideology, the sharing of data across HIEs and providers, and can equip healthcare providers with a holistic view of a patient's medical history.
\end{abstract}

Keywords: health information exchanges, HIEs, health information systems, health IS, patient access number (PAN), health information technology, health IT, electronic health records, e-Health

\section{Introduction}

Health Information Exchanges (HIEs) link healthcare providers by facilitating the transfer and storage of patient medical records [1] The Federal Agency for Healthcare Research and Quality (FAHRQ), reported that there are over 280 HIEs across the United States (U.S.). FAHRA further stated over 50 percent of hospitals utilize HIEs [2]. The major benefits of being a part of HIEs are: to coordinate and streamline patient care to reduce duplicate treatments; provide a basic level of interoperability among Electronic Health Records (EHRs) maintained by individual practitioners; provides a vehicle for improving quality and safety of patient care by reducing medication and medical errors [1].

The consensus among healthcare stakeholders is that HIEs play a critical role in improving the quality and efficiency of the healthcare system. To date, the U.S. has had limited success establishing broad base HIE systems. Healthcare provider support, financial sustainability, interoperability among HIEs and interconnectivity between HIEs are four major barriers that impede the progression of HIEs. Without strong support from healthcare stakeholders or implemented incentives, HIEs struggle to gain sufficient support for broadening exchange capabilities [3]. Interoperability or interconnectivity among certain medical technology, such as medical records, diagnostic tools, transcription, and security, is a critical issue for transitions of care [4]. Transition of care refers to the movement of patients between healthcare locations, providers, or different levels of care within the same location as their conditions and care needs change. This lack of interoperability decreases the value of HIEs which causes added costs, erosion of quality and timeliness of intervention [4].

\subsection{History and Evolution of HIEs}

+ Corresponding author.

E-mail address: dalvinhill@hotmail.com 
HIEs enable doctors and other medical practitioners to have access to patient medical history as is denoted in Figure 1, which is stored and retrieved from a centralized database. HIEs assist with timely information retrieval of patient treatment, supporting better quality of medical reports, public health activities, and assist with clinical research [5]. HIEs have real time data storage and retrieval for healthcare professionals to provide efficient patient care [6]. EHRs are stored in HIEs, and allows for quicker retrieval of more complete patient information by physicians and other providers [7]. Electronic lab reporting and prescribing within the HIEs can assist the Department of Health (DOH) and providers in identifying specific diseases, such as tuberculosis and viral hepatitis, before they affect a significant part of the population [8]. The healthcare system can be revolutionized by the enhancement and evolution of HIEs.

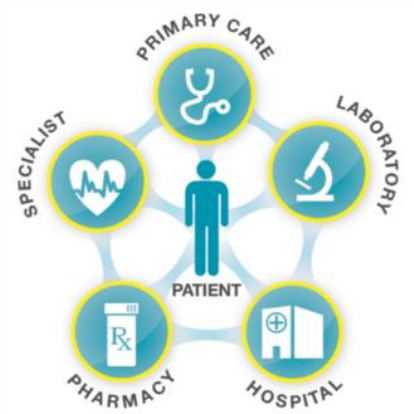

Fig. 1: (HIE Benefits, 2014).

For over twenty years, there have been efforts to establish HIEs in the U.S. In 1990, the Harford Foundation created the Community Health Management Information System (CHMIS). The CHMIS established a centralized medical repository in seven distinct geographical communities [9]. In the mid 1990's, Community Health Information Networks (CHINs) evolved and allowed healthcare providers to share patient data in a more cost-effective manner [9]. Additionally, in 2004, the U.S. Departments of Defense (DOD) and Veteran Affairs (VA) began working on an exchange system of medical records for military personnel [10].

In 1998, the DOD and VA created initiatives to exchange patient information [11]. To date, both departments' initiatives include: (1) one-way transfer of discharged service members' EHRs from the DOD to the VA through the Federal Health Information Exchange (FHIE); (2) EHR of shared patients from both departments can be accessed through the Bidirectional Health Information Exchange (BHIE); (3) the DOD and VA can exchange computable outpatient pharmacy and drug allergies information through the Clinical Data Repository/ Health Data Repository (CHDR); and (4) the departments can share laboratory information through the Laboratory Data Sharing Interface (LDSI) [11]. With these initial developments of HIEs, and with the passing of key legislation, there has been a promotion in the development and growth of HIEs across the U.S.

\subsection{Federal Legislation and Funding}

The development of HIEs is a key concept of the federal government for a national healthcare information network [5]. In 2004, President Bush established the Office of the National Coordinator for Health Information Technology (ONC), which sets a foundation of interoperability initiatives for EHRs [12]. These ONC initiatives consist of five major protocols that includes: The American Health Information Community, Standards Harmonization Process, Compliance Certification Process, Privacy and Security Solutions, Nationwide Health Information Network Prototypes [12]. The ONC is a resource of support for the healthcare system in adopting Health Information Technology (HealthIT). On February 13, 2009, due to the economic crisis and at the urging of President Obama, Congress passed the American Recovery and Reinvestment Act (ARRA) which included $\$ 44$ billion for EHRs, data connectivity and the development of privacy and security standards [13]. The directive of the 2013 Affordable Care Act (ACA) was for states, or the federal government to create HIEs. The health insurance exchange would then rely on HIEs to determine eligibility and provide transparency about insurance pricing and quality of care [10]. As the HIE market evolves, two emergent models prevailed: public HIEs and private HIEs. 
The development of HIEs to store EHRs can be attributed to Federal mandates and initiatives from the private sector. HIEs enable healthcare providers with real time access to patients' medical history. However, even with the creation of HIEs, there still exists a siloed effect of medical records. The passing of the ACA provides an opportunity for the Federal Government to control the standardization of HealthIT and an opportunity to move toward interconnectivity of HIEs. The ACA and HealthIT have established three meaningful use stages for providers to accomplish an improvement in healthcare for the population [14]. As it relates to interconnectivity, stage one focuses on standardizing electronic health records; stage two pertains to electronic transmission of EHRs over multiple settings; stage three emphases the ability to access EHRs from a patient centralized HIE.

\subsection{Sustainability of HIEs}

Due to reforms and initiatives in the healthcare system, healthcare providers are integrating patients' EHRs with either public or private HIEs [15]. Healthcare providers can choose public HIEs that use government funding, or private HIEs where investments come from stakeholders or private healthcare organizations. Title XIII- HITECH of the ARRA, allotted \$300 million to support the development of HIEs [13]. States receive funding from HITECH and other sources to construct HIEs or upgrade existing ones. Due to the discontinued funding by the federal government at the end of 2014, some HIEs struggle to survive [16].

There are barriers that need to be overcome in order for HIEs to be more prosperous. The major barriers that are affecting the success of HIEs include privacy/security and sustainability. Inadequate security measures have led to medical database breaches and this in turn led to patient concerns of electronic storage and sharing of EHRs [17]. There are obstacles for HIEs to maintain sustainability and challenges of maintaining infrastructure for ongoing interoperability. There is a lack of financial incentives for stakeholders to invest in HIEs. Also, organizations that take on fiscal responsibility are ensured benefits from the services of HIEs [16].

\subsection{Benefits of HIEs}

HIEs store medical information in the form of EHRs. With authorized access to HIEs, providers are able to review up to date patient medical history. This is particularly significant for patients who are seeing multiple specialists, making transitions between health facilities, or receiving emergency care [18]. In a 2013 study conducted by the U.S. Department of Health and Human Services (HHS), it was demonstrated that HIEs have resulted in: reduced hospital admissions, avoided emergency department transfers, and improved physician follow-up care after discharge [19]. HIEs foster better availability of patient information which can ultimately reduce medical errors and unnecessary tests [18].

Healthcare providers that have access to patient's medical history can eliminate the need for duplicate tests or scans. This relates to more efficient treatment as it saves time required to complete repeat tests and reduce the cost of medical care [20]. A study, conducted by University of Michigan Medical School (UMMS), showed that emergency room patients received fewer repeat tests or scans when hospitals were networked within HIEs [21]. Dr. Keith Kocher, research author and U-MMS emergency room physician stated that emergency departments were chosen for the study due the urgency of care [20]. Emergency departments need a patient's medical history quickly in order to expedite diagnoses and treatment options. The data in the study was collected from emergency rooms in California and Florida which are two of the larger states that were among the early adopters of HIEs. The findings showed that the use of repeat chest Xrays, CAT and ultrasound scans were significantly lower when patients had both their emergency visits at two unaffiliated hospitals that subscribed to a common HIE [20].

In a paper-based healthcare system, it can be time consuming, expensive, and inconvenient for an individual to get copies of his/her medical records. For patients that require specialized care, there needs to be improved coordination of information exchange among the patients' healthcare providers [22]. With the development of eHealth tools and the VA's Blue Button, individuals have access to pertinent medical information. The VA Blue Button feature is an online patient portal that allow patients to easily download their personal health information [22]. The eHealth devices such as digital scales and wireless pedometers, 
help patients keep track of certain health metrics. Mobile device applications provide information to online communities to help individuals interpret the medical data, receive emotional support, and make choices that support personal health goals [23]. Patients can use their health information to better communicate with providers and peers, better understand health and treatment options, and verify their health information is as accurate and complete as possible [23]. These virtual healthcare tools can assist healthcare providers monitor their patients' health. This information in turn can also be added to the patients' EHRs which is stored in the HIE system.

\subsection{Drawbacks of HIEs}

There are major concerns of privacy rights and data security affiliated with the electronic transmission and storage of EHRs [17]. The lack of adequate security measures have resulted in numerous data breaches, leaving patients exposed to economic threats, mental anguish, and possible social stigma [17]. If the public cannot trust that their health information will be both private and secure, they may not seek the help they so rightfully need.

Hospitals and other healthcare professionals have reservations as it pertains to integrating EHRs into HIEs. Since HIEs allow authorized access to EHRs, healthcare professionals fear losing patients to other providers who are a part of the HIE. A healthcare professional can differ with a treatment or diagnosis and sway a patient to go with their professional opinion. One of the greatest apprehensions of healthcare providers is controlling who has access to patient data. Hospitals fear that competitors or other parties can use certain data to harm the hospital's business interests. They claim these competitors will use this data as a marketing ploy to redirect patients to other providers [5]. Providers are not only concerned with the cost implementation of EHRs, but the repercussions if they do not achieve "Meaningful Use" of certified EHRs in order to receive financial incentives [14]. HITECH defines Meaningful Use as the ability to use the EHR to effectively support specific clinical activities [24]. Also, to avoid penalties to Medicare rates, eligible healthcare providers must be engaging in meaningful use by 2015[14]. A major drawback to HIEs is that it has not completely solved the issue of fragmentation of patient medical records that exist within the various HIEs.

\section{Proposed Solution}

With over 280 HIEs in the U. S., there needs to be a way in which they can all communicate with each other and share data. Failure to do so will result in fragmentation of patient data, similar to paper based records, or even siloed EHRs. This paper aims to find a solution for the interconnectivity of HIEs that will ultimately foster the sharing of patient data while reducing the fragmentation. There have been national and state level governance efforts to standardize HIEs. A variety of national efforts have been put forth but no uniform standards have been adopted. States have followed different pathways when it comes to governing HIEs. States have either developed statewide, community based or regional policies to govern their HIEs [25]. The federal government has incentives to encourage the development of discrete delivery of data for entities that are related to one another and this approach is referred to as push or direct exchange. Others support the idea of authorized users having the ability to query data from multiple systems which is referred to as "pull" or query exchange [25]. As it relates to uniform health information infrastructure systems, there are fundamental standards that are recognized. These standards are implemented on individual HIE systems, but the inconsistencies exist between pairs of HIE institutions that wish to exchange patient information [26].

The lack of interconnectivity among HIEs is part of the missing link to bring quality healthcare for Americans to the national level. The barrier of interoperability and interconnectivity among HIEs are being addressed and solutions are being developed. A paper written by Dr. Dalvin Hill et al. suggests the issuance of a Patient Access Number (PAN) for all U.S. citizens and residents [27]. This standard for identifying patients is one viable step to the solution of interconnectivity between HIEs. The goal of this paper aims to concatenate the solution proposed by Hill, et al. with hopes of enhancing the interconnectivity of HIEs. A careful analysis was conducted for the proposed solution and it incorporates a hybrid approach emulating aspects of law enforcement systems along with a PAN [27]. The National Crime Information Center (NCIC) system and the research behind the PAN proposal are proven schemes that work. HIEs can be optimized by 
these proposed systems to develop interconnectivity. By concatenating both approaches, we are moving a step closer to solving the siloed data of HIEs.

Law enforcement agencies across the U.S. have access to a local and a national crime database [28]. The database infrastructure has security and policies in place to uphold data integrity, and authentication. This came about in the 1960s when FBI Director J. Edgar Hoover and the International Association of Chiefs of Police decided to implement a computer system that would centralize crime information. When the NCIC system was initially launched in January 1967, it had five categories and approximately 356,000 records [28]. This system incorporates crime information from every state and allows law enforcement agencies across the nation access [28].

At the local level (i.e. each state), there is a localized system/database. For example, in the state of Texas, there is the Texas Crime Information Center (TCIC) [30]. TCIC can be accessed by law enforcement agencies throughout the Texas Law Enforcement Telecommunications System (TLETS) to return results of driver's license information, stolen items or information about persons who are wanted [30]. This data can also be entered and accessed on the national level. Through the National Law Enforcement Telecommunications System (NLETS), law enforcement agencies can access the NCIC database to broaden a query on a person, or possible stolen item they have detained for investigative purposes [31]. These systems, TLETS and NLETS have been around since 1960s and have proven to be reliable [31]. It is inevitable that much can be learned from examining the aforementioned law enforcement systems. Figure 2 shows the hierarchy of law enforcement's criminal information database system.

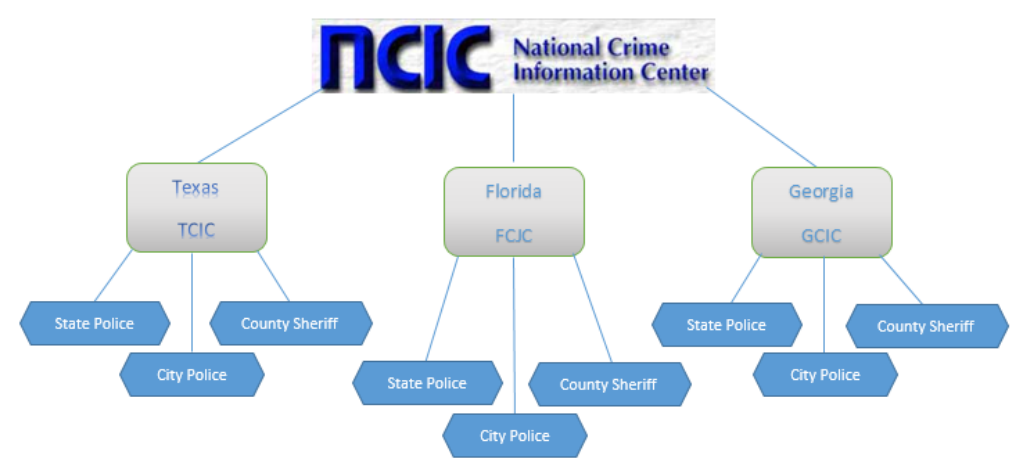

Fig. 2: Hierarchy structure.

The NCIC system uses a two prong management concept - policy and functional. The Criminal Justice Information Services (CJIS) Advisory Board provides the Meaningful Use policy for agencies that access NCIC. This Board permits NCIC users to make recommendations to the FBI Director for policy and operational enhancements to the system. The functional concept is the communication medium used to access the NCIC. The FBI provides a main computer and telecommunication lines, NLETS, to a single point of contact to each state (for example: TCIC in Texas). Each state has its own criminal database, and each agency of said state is responsible for entry, modification and removal of records. CJIS Division of the FBI serves as a custodian over NCIC and the records it contain. Today, NCIC system contains 21 categories and close to 12 million active records. The NCIC database averages around eight million transactions a day with an approximate response time of 0.1119 seconds per transaction [29]. Cooperation and shared management concept between the FBI, federal, state, local, and tribal criminal justice users is essential and are the keys to NCIC's success.

The CJIS System Agency is responsible for security and quality control. The CJIS System Officer (CSO) is appointed by the head of the CJIS and is responsible for monitoring system use, enforcing system discipline and security, and assuring that all users adhere to standard operating procedures [29]. To ensure the privacy and integrity of data, NCIC establishes a number of security policies. Data passing over the lines of communication are highly encrypted to deter unauthorized access. For every transaction query, each user is authenticated to ensure proper level of access. Agencies must also undergo periodic audits to ensure data quality and adherence to all security procedures. In addition to studying the efforts of the law enforcement 
agencies, the work proposed by Hill et al. [27] might be a part of the solution. The Patient Access Number (PAN) would be issued to Americans by the HHS, and can be used by healthcare providers to access medical records. The PAN is an eleven-digit number comprised of a two-digit state code, and the remaining nine digits is an auto generated unique number as denoted in Figure 3. This nine-digit auto generated number may be duplicated in each state. However, adding the two-digit state code and enforcing data integrity prevents any two patients from having the identical 11-digit PAN. A PAN would ensure the unique patient information is retrieved. PAN is a unique number of an individual whereas names, birth dates, address, and phone number may not be. The less data a healthcare provider has to use to identify a patient, the more room for error and as such, PAN is an ingenious solution to link a person with their medical history.

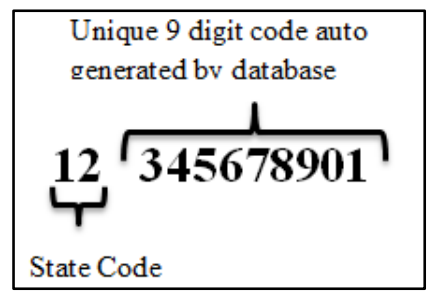

Fig. 3: PAN [27].

The proposed solution is to emulate the interconnectivity and interoperability that the NCIC has established with each state. The federal HHS will manage the database to enforce data integrity so that no two individuals would receive a duplicate PAN. Each PAN record in the database will have information associated with it such as name, date of birth, address, SSN, insurance company name along with group and member ID number. Based on this proposal, the federal HHS will create a centralized database for HIEs - to be called the National Health Information Exchange (NHIE). The NHIE database for medical records is synonymous to the FBI's NCIC and will have a central point of contact for each state and territory of the U.S. For example, if you are a Texas resident visiting Florida and are in need of medical attention, a healthcare provider in Florida would be able to access your medical history using your PAN. All data passing to and from these telecommunication lines will be highly encrypted to reduce security breach or unauthorized access. The federal HHS will enact and enforce strict regulations and standard operating procedures. These procedures are to ensure patients' privacy rights are not violated and accurate medical information is documented. Figure 4 shows the proposed levels of infrastructure from the national level down to the healthcare providers. The National HIE is to be monitored by the federal HHS and branches out to each State HHS forming the State HIE (SHIE). The SHIE links the healthcare providers HIEs so that hospitals, doctors, clinics and pharmacy are all interconnected.

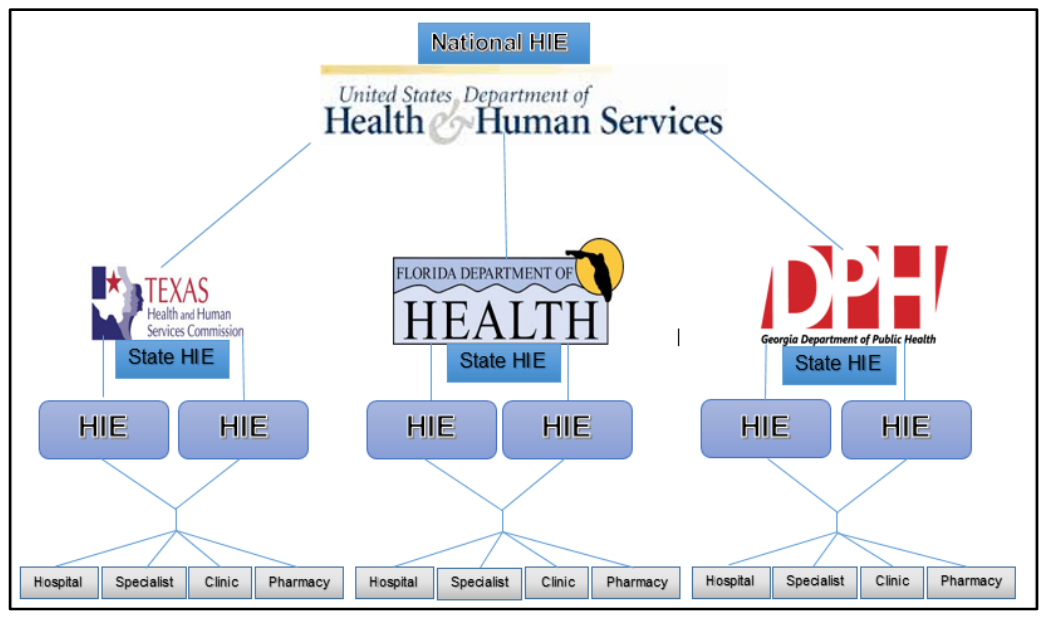

Fig. 4: Proposed HIE interconnectivity.

The main problem with HIEs is that they are not all interconnected with one another. Even if individual HIEs used the same vendor that designed their systems, it does not guarantee they would have 
interconnectivity between one another. To solve the interconnectivity issue, the federal HHS needs to create network protocols so that the NHIE will interconnect with all SHIEs. Each state's HHS agency will be the point of contact for the central telecommunication line to the NHIE. The HHS of each state will oversee the development and growth of their HIE network which can be compared to the TCIC network for Texas crime records. This will ensure the network protocols set forth by the federal HHS are adhered to not only by HIEs, but also by healthcare providers that have access them.

Healthcare professionals need to have systems in place that support secured two-way traffic with the HIEs. They need to have real time access to patients' medical records and be capable of updating a person's EHR similar to updating a record in the TCIC and or the NCIC. The update needs to be instantaneous so transition of care is seamless and without delay. If a patient is outside their healthcare network and needs to see a healthcare professional, this should not be a hindrance on efficient medical service; neither should it create a siloed medical record. With the interconnectivity of all HIEs (both locally and nationally), the healthcare provider will be able to search for a patient's medical information using their PAN, since it is unique. This will in turn give the patient efficient and quality treatment since the medical record will now provide a holistic view of diagnosis and treatment.

\section{Conclusion}

Americans deserve a healthcare system that is reliable, efficient, and affordable. As the name implies, the Affordable Care Act of 2009 is striving to make healthcare affordable by implementing policies that will streamline certain procedures of the healthcare system. Patients need to be assured that when they walk into any medical facility, the staff can provide optimal healthcare treatment. By having real time access to patient's medical history through interconnected HIEs, it will enable healthcare providers to diagnose and treat patients more efficiently.

This paper proposes an idea for an enhanced database system that will provide interconnectivity for all HIEs. The idea replicates the system that the FBI's NCIC use to collect and store crime information records from each state. Through the federal HHS, a national healthcare exchange database would serve as the storage facility for every American's medical record. The HHS's NHIE database will ensure data integrity by issuing every person a PAN. The proposal of the law enforcement's model coupled with the PAN will solve the HIE interconnectivity issue. This will meet the government's objective for a national healthcare system that is referenced in Stage 3 of the Meaningful Use. With the interconnectivity of HIE's, patients' EHRs can be accessed and updated in real time by healthcare providers. The ability to have real time visibility results in efficient patient care that eliminates the redundancy of medical tests and scans. Eliminating repeat procedures will result in savings for both the patients and healthcare providers. Real time access to patient medical records allows healthcare providers to make a quicker diagnosis which puts the patient on a faster road to recovery, and can ultimately reduce hospital stays. On an economical level, the patients, insurance companies, and healthcare providers will save money. Fast efficient healthcare will reduce out of pocket expense for the patient; while there will be fewer pay outs by insurance companies and a reduction in the expenditures of hospital resources. Interconnectivity of HIEs is the key concept for resourceful healthcare in America.

\section{References}

[1] HIE Benefits. (2014, march 14). HIE Benefits. Retrieved from healthit.gov: http://healthit.gov/providers-professionals/health-information-exchange/hie-benefits

[2] Hagland, M. (2013, August 30). Health Information Exchanges: Are We At An Infection Point? Retrieved from Healthcare Informatics: http://www.healthcare-informatics.com/article/healthinformation-exchange-are-we-inflection-point

[3] Prashila Dullabh, M. (2014, Aug). Key Challenges to Enabling Health Information Exchange and How States Can Help. Chicago, Ill, US.

[4] NTOCC. (2010, Dec http://www.ntocc.org/portals/0/pdf/resources/hitpaper.pdf). Improving Transition of Care With Health Information Technology. 
[5] Grossman, J. M. (2008, 2 2). Creating Sustainable Local Health Information Exchanges: Can Barriers to Stakeholder Participation be Overcome? p. 12. Retrieved 2015, from http://www.hschange.org/CONTENT/970/970.pdf

[6] HealthIT.gov. (2013, Aug 20). Accelerating Health Information Exchange (HIE). Retrieved from healthit.gov: http://www.healthit.gov/policy-researchers-implementers/accelerating-health-informationexchange-hie

[7] NCHICA.org. (2015). FAQs About Electronic Health Records and Health Information Exchange. Retrieved from nchica.org: http://www.nchica.org/GetInvolved/CACHI/FAQs.htm

[8] Goldwater, J. (2014, 09 23). Emphasizing Public Health Within a Health Information Exchange: An Evaluation of the District of Columbia's Health Information Exchange Program. EDM Forum-EDM $\begin{array}{llllll}\text { Forum } & \text { Community. } & \text { Retrieved } & 02 & 12, & 2015,\end{array}$ http://repository.academyhealth.org/cgi/viewcontent.cgi?article=1090\&context=egems

[9] Dullabh. (2011). The Evolution of State Health Information Exchange Cooperative Agreement Program: STATE PLANS TO ENABLE ROBUST. Retrieved from https://healthit.gov/sites/default/files/CaseStudySynthesisGranteeExperienceFinal_121014.pdf

[10] Scott, D. (2012, Feb 09). Health Information Exchange Implementations Encounter Obstacles. Retrieved from governing.com: http://www.governing.com/blogs/view/health-information-exchangeimplementations-encounter-obstacles.html

[11] Panangala, S. V. (2013, 02 26). Departments of Defense and Veterans Affairs: Status of the Integrated Electronic Health Record (iEHR). Retrieved 02 11, 2015, from https://www.fas.org/sgp/crs/misc/R42970.pdf

[12] Cronin, K. (2015). Office of the National Coordinator for Health Information Technology. Retrieved from www.deadiversion.usdoj.gov: http://www.deadiversion.usdoj.gov/ecomm/e_rx/mtgs/july2006/kcroninpp.pdf

[13] ARRA. (2009). American Recovery and Reinvestment Act of 2009. Pub. L. 111-5, 407. United States. Retrieved 02 22, 2015, from http://www.gpo.gov/fdsys/pkg/BILLS-111hr1enr/pdf/BILLS111hrlenr.pdf

[14] Metzger, J. e. (2010). Meaningful_Use_for_Hospitals_The_Top_Ten_Challenges. Retrieved 0223 , 2015 , http://assets1.csc.com/health_services/downloads/CSC_Meaningful_Use_for_Hospitals_The_Top_Ten_ Challenges.pdf

[15] Manish, s. (2013, 10). Choosing between public and private HIEs. Retrieved from healthmgttech.com: http://www.healthmgttech.com/articles/201310/choosing-between-public-and-private-hies.php

[16] Bostick, et al. (2011). Sustaining State Health Information Exchange: A State Toolkit. Retrieved from: https://www.nga.org/cms/home/nga-center-for-best-practices/center-publications/page-healthpublications/col2-content/main-content-list/sustaining-state-health-informat.html

[17] Appari, A.,. (2010). Information security and privacy in healthcare: current state of research. Int. J. Internet and Enterprise Management, 6(4), 279-314. Retrieved 02 16, 2015

[18] Bell, B. T. (2011). Improved Care Coordination The Need for Better Improved Care Coordination. Retrieved from healthit.gov: http://www.healthit.gov/providers-professionals/improved-carecoordination

[19] Wood, D. (2014, 07). Health Information Exchange is a Key to Better Transitions, Better Outcomes and Cost Savings. Retrieved from leadingage.org: http://www.leadingage.org/HIE_Key_to_Better_Transitions_Better_Outcomes_and_Cost_Savings_V4 N4.aspx

[20] Garvin, K. (2014, 01). When hospitals share patient records, emergency patients benefit, study suggests. doi:10.1097/MLR.0000000000000067

[21] Lammers, E et al. (2014, 52 03). Does Health Information Exchange Reduce Redundant Imaging? 
Evidence From Emergency Departments. doi: http://10.1097/MLR.0000000000000067

[22] Turvey, Carolyn et al. (2014, 07 01). Blue Button use by patients to access and share health record information using the Department of Veterans Affairs' online patient portal. doi: http://dx.doi.org/10.1136/amiajnl-2014-002723

[23] Ricciardi, L. (2012, 06 25). Making Patient Access to Their Health Information a Reality. Retrieved from healthit.gov/buzz-blog: http://www.healthit.gov/buzz-blog/consumer/making-patient-accesshealth-information-reality/

[24] Battani, J. (2009). MEANINGFUL USE:. Retrieved 2016, from http://assets1.csc.com/health_services/downloads/CSC_Meaningful_Use_for_Health_Plans.pdf

[25] Manatt Health Solutions. (n.d.). Ten Years in: Charting the Progress of Health Information Exchanges in the US. California, US. Retrieved 01 26, 2015, from http://www.chcf.org/ /media/MEDIA\%20LIBRARY\%20Files/PDF/T/PDF\%20TenYearsProgressHIE. $\mathrm{pdf}$

[26] Halamka, J. J. (2005). Exchanging Health Information: Local Distribution, National Coordination. Health Affairs, 24(5). doi: 10.1377/hlthaff.24.5.1170

[27] Hill, D. D. (2015). Interconnectivity of Health Information Exchanges Using Patient Access Number. South University - Austin, Austin.

[28] FBI. (2015). National Crime Information Center. Retrieved from FBI.gov: http://www.fbi.gov/aboutus/cjis/ncic

[29] FBI. (2015). National Crime Information Center (NCIC). Retrieved from fas.org: http://fas.org/irp/agency/doj/fbi/is/ncic.htm

[30] DPS. (2015). Texas Crime Information Center (TCIC). Retrieved from txdps.state.tx.us: http://www.txdps.state.tx.us/administration/crime_records/tcic/

[31] NLETS. (2015). Who We Are. Retrieved from Nlets - The International Justice And Public Safety Network: http://www.nlets.org 